\title{
Cascade sensitivity studies for KM3NeT
}

\author{
Luigi Antonio Fusco ${ }^{1,2, a}$ on behalf of the KM3NeT Collaboration \\ ${ }^{1}$ Dipartimento di Fisica e Astronomia dell’Universitá, Viale Berti-Pichat 6/2, 40127 Bologna, Italy \\ ${ }^{2}$ INFN - Sezione di Bologna, Viale Berti-Pichat 6/2, 40127 Bologna, Italy
}

\begin{abstract}
KM3NeT is a future research infrastructure in the deep seas of the Mediterranean housing a large scale neutrino telescope. The first phase of construction of the telescope has started. Next step is an intermediate phase realising a detector volume of about one-third of the final detector volume. We report on calculations of the sensitivity of the KM3NeT detector to showering neutrino events, the strategy to optimise the detector to a cosmic neutrino flux analogous to the one reported by the IceCube Collaboration and the results of this strategy applied to the intermediate phase detector.
\end{abstract}

\section{Introduction}

KM3NeT [1] is the next generation neutrino telescope being built in the Mediterranean Sea. It will consist of several thousand Digital Optical Modules (DOMs) [2], pressure resistant glass spheres housing $313^{\prime \prime}$ photomultiplier tubes (PMTs). DOMs will be distributed along vertical strings, each holding 18 of them vertically spaced by $36 \mathrm{~m}$. Strings will be anchored to the seabed at an average distance of $90 \mathrm{~m}$ from each other. 115 strings arranged together will constitute a detector building block.

The final detector will consist of 6 building blocks, for an instrumented volume of about 3-5 $\mathrm{km}^{3}$, distributed at different locations in the Mediterranean Sea. The main aim for the KM3NeT Collaboration will be to search for cosmic neutrino sources, especially in the Galactic Region. The Phase 1 of the project is currently in its final development steps [3]. The first phase of construction of the detector has started.

The next phase of construction is an intermediate step to construct a detector of two building blocks. This phase of the detector ("Phase 1.5") will be dedicated to the confirmation of the isotropic and all flavor cosmic neutrino signal recently reported by the IceCube Collaboration [4]. This signal can be, for example, parametrised as

$$
\mathrm{E}_{v}^{2} \Phi_{v}=1.2 \cdot 10^{-8} e^{-\mathrm{E}_{v} / 3^{\mathrm{PeV}}} \mathrm{GeVs}^{-1} \mathrm{sr}^{-1} \mathrm{~cm}^{-2} .
$$

The observation of such a signal by an independent experiment, with different systematics using a different neutrino converting and light detection medium, would constitute an extremely important discovery.

\section{Event selection procedure}

Many of the neutrino events reported by IceCube look like shower events (cascades), generated by $\mathrm{NC}$ and $\mathrm{CC} v_{e}$ and $v_{\tau}$ interactions and $v_{\mu} \mathrm{NC}$ interactions. Cascades release

\footnotetext{
${ }^{a}$ e-mail: luigiantonio.fusco@bo.infn.it
} 

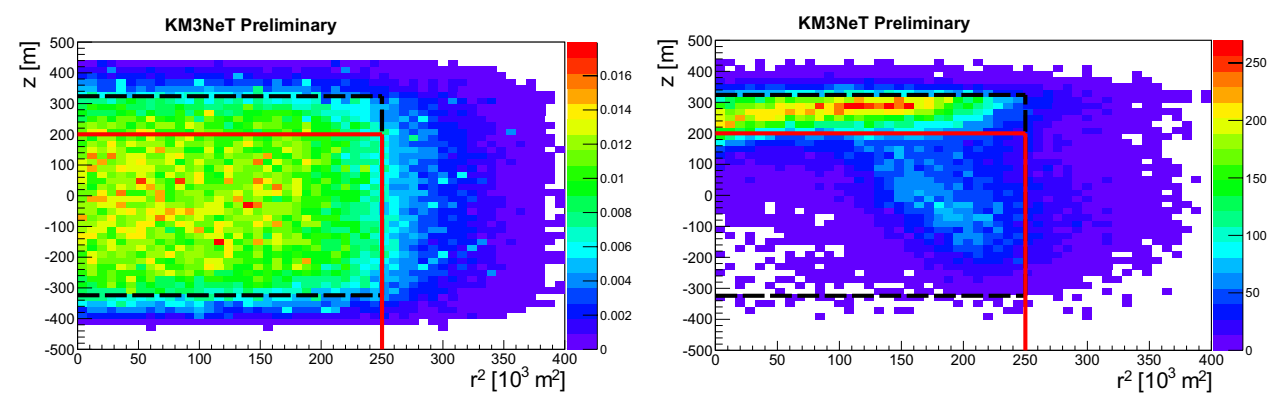

Figure 1. Output of the vertex reconstruction for signal cosmic neutrinos (left) and background atmospheric muons (right). The $\mathrm{z}$ and $\mathrm{r}^{2}$ coordinates (see text) of the reconstructed vertex are plotted. The black dashed line represents the instrumented volume of a KM3NeT detector building block, while the red solid line is the containment requirement for the analysis. This selection allows for a good muon rejection.

a large amount of light starting from the interaction vertex region and this signature can be properly reconstructed.

A large background of atmospheric events is expected when searching for cosmic neutrinos. Atmospheric neutrinos and muon bundles from air showers can reach large sea depths and mimic the signal. Background neutrinos can be rejected with a proper energy cut, as their steep spectrum lowers their contribution at high energy. Atmospheric muon rejection requires different strategies.

Four selection cuts for signal events have been defined using a set of simulated events reconstructed as cascades.

1. $\mathrm{N}_{\text {hit }}$ : more than 2000 PMT pulses are required. Below this threshold the signal to noise ratio is always negligible because of atmospheric events.

2. Containment: the distribution of reconstructed vertices for atmospheric muons shows an accumulation in the uppermost part of the instrumented volume. On the other hand, cosmic showers vertices are expected to be uniformly distributed in the detector (Fig. 1). As a consequence, cutting on the reconstructed vertex position improves the rejection of atmospheric muon bundles.

3. Time over Threshold: the Time over Threshold (ToT) of each PMTs, summed over all hits that are causally correlated with the reconstructed vertex, is used to discriminate events both by their topology and by their energy. A shower event is expected to have more selected hits than a track, leading to a larger sum of ToT; high energy events would be selected because of higher hit-wise ToT.

4. Boosted Decision Tree: a machine learning technique has been used as shower selector, trained with different quantities defined in the reconstruction algorithms for KM3NeT events. The output of this method is then used, together with the energy estimator $\rho$, in a two dimensional Model Rejection Factor minimization (MRF) [5], to obtain the final event sample (Fig. 2).

The resulting chain of cuts, maximising the sensitivity, is:

$$
\mathrm{N}_{\text {hit }}>2000 ; \mathrm{r}<500 \mathrm{~m} \text { and } \mathrm{z}<200 \mathrm{~m} \text {; ToT }>15 \mu \mathrm{s} ; \text { BDT }>0.35 \text { and } \rho>10^{6},
$$

being $\mathrm{r}$ and $\mathrm{z}$ the cylindric coordinates of the reconstructed vertex. 

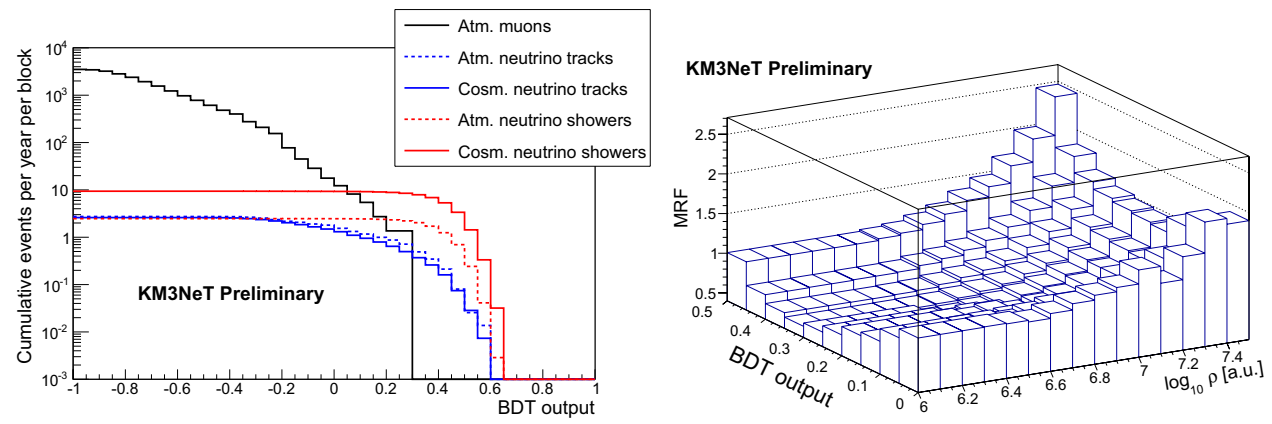

Figure 2. Output of the BDT application to every event type (left) - color code as in the legend. Application of the 2D MRF minimization with the BDT output and the energy estimator $\rho$ (right). The minimum is found at $\rho>10^{6}$ and BDT $>0.35$, the selection cut maximising the sensitivity [5].
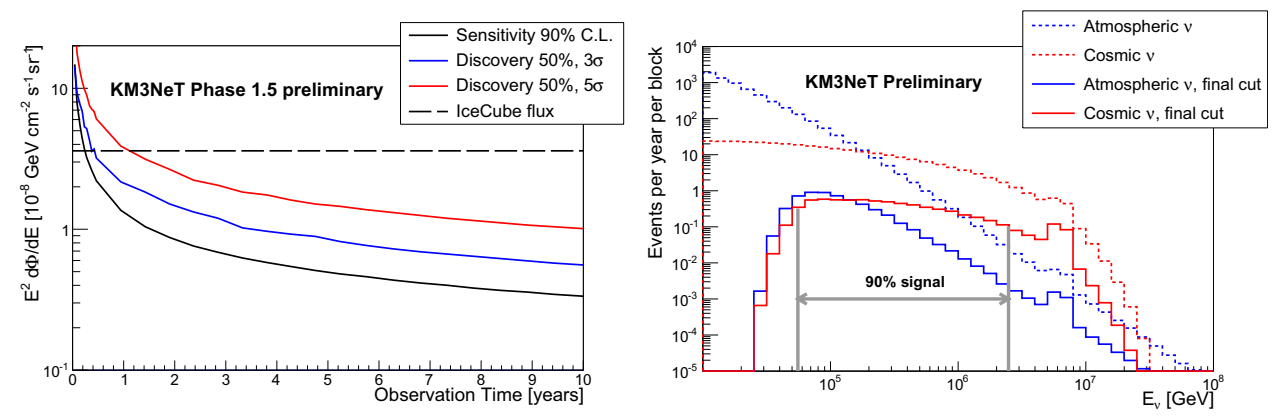

Figure 3. Left: $90 \%$ C.L. upper limit sensitivity and 3 and $5 \sigma$ discovery potential for the detector of KM3NeT Phase 1.5 to the flux of Eq.1, color code as in the legend. Right: energy distribution of signal and background events after selection cuts; the central $90 \%$ of the signal lies between $56 \mathrm{TeV}$ and $2.5 \mathrm{PeV}$.

\section{Results}

The background is extremely reduced after the cut optimization, as 6.7 signal events are expected to be detected on top of a background of 2.2 per year per detector building block: the performance of the detector is consequently very promising.

After the MRF procedure, the 90\% C.L. sensitivity flux for the cosmic neutrino flux reported in [4] is obtained. The KM3NeT Phase 1.5 detector would have the possibility to probe an IceCube-like signal in a short time. As shown in Fig. 3, this limit is valid over and energy range between $56 \mathrm{TeV}$ to $2.5 \mathrm{PeV}$. Using the optimized selection cut, the $3 \sigma$ and $5 \sigma$ significance for a $50 \%$ discovery can be calculated, and the evolution with observation time of the detector performance is shown in Fig. 3; with the neutrino detector of KM3NeT Phase 1.5 it will be possible to discover a cosmic neutrino flux similar to the IceCube signal with a $5 \sigma$ significance in less than 1.5 years.

\section{References}

[1] U. Katz for the KM3NeT Collaboration, AIP Conf. Proc. 1630, 38-43 (2014)

[2] S. Adriáąn-Martinez et al. (KM3NeT Collaboration), EPJC 74, 3056 (2014)

[3] S. Biagi (for the KM3NeT Collaboration), these proceedings

[4] M.G. Aartsen et al. (IceCube Collaboration), Phys. Rev. Lett. 113, 101101 (2014)

[5] G.C. Hill \& K. Rawlins, Astrop. Phys. 19, 393 (2003) 